\title{
The plant diversity and conservation status of the Taurus and Amanos mountains in the Eastern Mediterranean Region of Turkey
}

\author{
Necattin Türkmen \\ Department of Biology, Faculty of Sciences and Letters, University of Cukurova, 01330 Adana, Turkey; e-mail: nturkmen \\ @)cu.edu.tr
}

ABSTRACT

In this study, the Taurus and Amanos mountains of Turkey's eastern Mediterranean region offer an overview of the floristic features. A total of 1,259 vascular plant taxa belonging to 106 families and 469 genera were recorded from the research area as a result of various researches and field studies conducted between 1999-2017. The phytogeographic elements were represented in the study as follows: Mediterranean 328 (26.1\%), Irano-Turanian 157 (12.5\%), Euro-Siberian 87 (6.8\%), cosmopolitan and unknown 687 (54.6\%). The total number of endemic species was 195 and the endemism rate was $15.1 \%$. According to the IUCN Red List Categories, 195 threatened plant species were found in the study area. As in all mountainous environments, climate, soil and vegetation characteristics change at short distances and are less exposed to anthropogenic activities. As a result, mountains are centers of high endemism and global biodiversity.

KEY WORDS Amanos; endemism; Taurus; flora; threatened species.

Received 14.10.2018; accepted 03.12.2018; printed 30.12.2018; published online 07.01.2019

\section{INTRODUCTION}

Very high parts of the earth's ridges (more than $500 \mathrm{~m}$ ) are called mountain, spreading over a very wide area with a steep slope, very difficult to climb, covered with trees, shrubs, grasses or bare rocks. Just as mountains can be isolated, they can form mountain chains, mountain ranges or mountain masses in relation to each other. Mountains are the forms of the earth which are meters above the earth and which are not suitable for human life and agriculture due to the generally harsh weather conditions. There are many mountains in the world and their origin is different. While some mountains are formed by the compression of the earth, some mountains come out to the surface due to lava and freeze. The source of the lava of the volcanoes is a very warm mass called magma. 54\% of Asia, 36\% of North America, 25\% of Europe, 22\% of South America, $17 \%$ of Australia and 3\% of Africa are covered with mountains. $24 \%$ of the Earth's terrestrial mass is entirely mountainous. Most of the world's rivers are fed by mountainous resources and are dependent on the mountains for more water than half of mankind. Mountains host approximately one quarter of all terrestrial biodiversity and half of the world's biodiversity hot spots (Anonymous, 2018; Barrow, 2018; Pariona, 2018).

Flatlands are generally uniform in vegetation. So a group of few plants can be seen. On the other hand, rugged terrain is rich in vegetation because it creates different environmental conditions. The 
forms of the earth especially affect the elevation, the direction of the mountain's extension, the unevenness, the spread of the plants and the formation of soil (Atalay, 2002, 2006; Özkan, 2008).

A large part of the forest areas in Turkey consists of natural forests. These areas, unspoilt by foreign species, have spread up to of mountainous masses. Turkey's mountains are also very rich in endemic plants at the same time.

In terms of genetic resources, mountain ecosystems have numerous important species, and, particularly, the mountains of Turkey have many endemic herbaceous species of the Anatolian Region (Öztürk et al., 2008). For this reason, mountainous areas have an extremely important function in agricultural biodiversity.

Anatolia is a geologically young mass. However, it is one of the most active centers in all geological periods. Due to intense volcanic and tectonic activity, different environmental conditions have led to the formation of high structures in different forms (single mountains, mountain ranges, and massive mountains) in the mountainous system. In ancient Western literature, Anatolia is referred to as Asia Minor because of its high altitude.

The Alpine-Himalayan mountain belt, which started about 65 million years ago (alpine period) and formed the youngest mountain formation zone in geological terms, is the rugged mountainous area (Anonymous, 2010). Mountain ranges in Turkey are also located in this mountain belt. Twenty-four percent of the Earth's land surface consists of mountainous areas. Approximately, $78 \%$ of Turkey's national area consists of mountain areas (Anonymous, 2010, 2011). In all mountain areas, elevation, climate, soil, and vegetation characteristics change at short distances. The climate varies depending on the latitude in the horizontal direction and the elevation in the vertical direction.

Turkey has, in general, the Mediterranean macro-climate. However, there are many different types of climate, depending on the shape of the ground (Erinç, 1993). Mountainous areas create isolated environments separating from the surrounding area, resulting in increased vegetative cover and locally distributed species.

Places with different bedrock, terrestrial and soil characteristics under the influence of the same climate type show distinct ecological characteristics (Kantarc1, 2005).
Differences in soil types play a crucial role in the diversity of plant communities and in the identification of their distribution area (Avc1, 2005).

Since the early ages, humans have been in close contact with plants (Baytop, 2000). Mankind has a wide variety of interventions directly and indirectly in the ecosystem they live in. Anthropogenic effects are sometimes more important than the sum of all other ecological factors (Duran, 2013).

Mountainous areas usually have low population density. Much of these areas are not suitable for human settlement. Plant communities in mountainous areas are also less exposed to anthropogenic effects. Plant diversity is uniform in flat areas where interaction is intensive (such as field opening, grazing, fire), open to human and animal interventions. The large increase in the world population has made an impact on vegetation in the natural environment.

Because of the high level of physical isolation in high mountain peaks, deep river valleys, closed basin lakes and karstic pits, only a few species are endemic to these areas (Atalay, 2002; Eken \& Ataol, 2011). Across the European continent, Turkey is one of the forested country with the highest rate (21\%) (Anonymous, 2010).

In high-altitude ecosystems, precipitation, wind, evaporation, and direct solar radiation increase. However, temperature, oxygen, atmospheric pressure, and nitrogen mineralization are reduced (Güleryüz et al., 2010). In addition, different ecological characteristics of the northern and southern slopes are very different from each other, so different plant breeds are formed (Atay et al., 2009). Mountain flora survive under different environmental conditions due to their adaptive properties (hairiness, dwarfness, needle leaflet, deep root, hard leaf, cluster formation, etc.) (Kılınç \& Kutbay, 2004).

Geographical isolation in mountainous areas, tectonic movements, climate changes, glacialization, strong microhabitat facilites, and evolutions increase the taxonomic richness (Körner, 1999).

In mountainous regions, rainfall, wind, air humidity, and direct radiation increase as height increases, while temperature, water vapor, and air pressure decrease and vegetation duration shortens (Duran, 2013).

The micro-habitats that characterize the mountainous areas vary according to the roughness, elevation, and slopes of the land. Due to the interactions between soil, temperature and surface, 
which vary with the effect of the sun and wind, very large changes are observed at very short distances. With the effects of these changes in micro-habitats, the mountain belts contain a very rich biodiversity (Atay et al., 2009).

Many mountain ecosystems have higher species richness and endemism levels than adjacent plains. The mountains at lower altitudes can support an extraordinary biodiversity because a wide variety of ecosystems are compacted to a relatively short distance. The mountains also have suitable living areas isolated from unsuitable low plains.

Endemism levels are high, especially in the tropical regions and in the medium-height mountains in temperate regions. Mountains show environmental changes for some migratory species or represent refuge areas for other species. Mountain species with low habitat tolerance, especially those of high-altitud, are at a higher environmental risk to climate change.

Mountain environments cover $27 \%$ of the world's land surface and directly support $22 \%$ of the world's people living in mountain regions. The people of the valley are also dependent on mountain environments for a wide range of products and services, including water, energy, timber, biodiversity care, recreation and spiritual renewal opportunities. The mountains satisfy the freshwater needs of more than half of mankind and are actually the world's water towers. The mountains of the world are made up of the most spectacular landscapes, from a wide variety of species and habitat types and from diverse human communities. Mountains all over the world, in all latitudes, from hyper-arid warm desert to tropical forests, support a wide variety of ecosystems. Mountain ecosystems are important for biodiversity, especially in tropical regions and warm temperate latitudes. Isolated mountain blocks are often rich in endemic species.

In this study, the Taurus and Amanos mountains in Turkey's eastern Mediterranean region was chosen to determine the overall plant diversity and conservation status.

\section{MATERIAL AND METHODS}

\section{Study area}

The research area within the borders of Adana and Hatay province covers the geographical moun- tainous area within the boundaries of Erzin, Dörtyol and Iskenderun districts of the central Amanos mountains and the geographical mountainous area within the Kozan and Feke district borders of the central Taurus mountains (Fig. 1). Main settlements in the research area are Mansurlu, Burhaniye, Kalkumaç, Ergenuşağı, Bahçecik, Topaktaş, Karıncalı, Başlamış, and Üçkoz villages. Important rivers in the study area are the Zamantı and Göksu rivers, and there are İnderesi, Özerli, Deliçay and Payas creeks. The most important mountains in the study area are Tahtaflatan $(2,495 \mathrm{~m})$, Saripinar $(2,081 \mathrm{~m})$, Karakızoluğu $(2,158 \mathrm{~m})$, Sicimindağ 1 (2,259 m), Görbeyaz (1,948 m), Mığır (2,240 m), and Harmankaya (2,208 m) (Fig. 2).

The Taurus and Amanos mountains, where the study area is located, constitute an important part of the Alpine orogenic belt that passes through the southern and eastern parts of Anatolia (Özgül, 1976). Geologically, the study area is formed from Mesozoic and Cretaceous limestone, upper Cretaceous ultrabasic rocks (Gabro and Serpentine), and Tertiary marls. Common soil formations distinguished in the area are as follows: brown calcareous soils, brown forest soils, terrarosa soils, reddishbrown Mediterranean soils, colluvial soils, and mixed land types (Akman, 1973; Türkmen \& Düzenli, 1990; Karakuş \& Türkmen, 2014; Türkmen et al., 2015a).

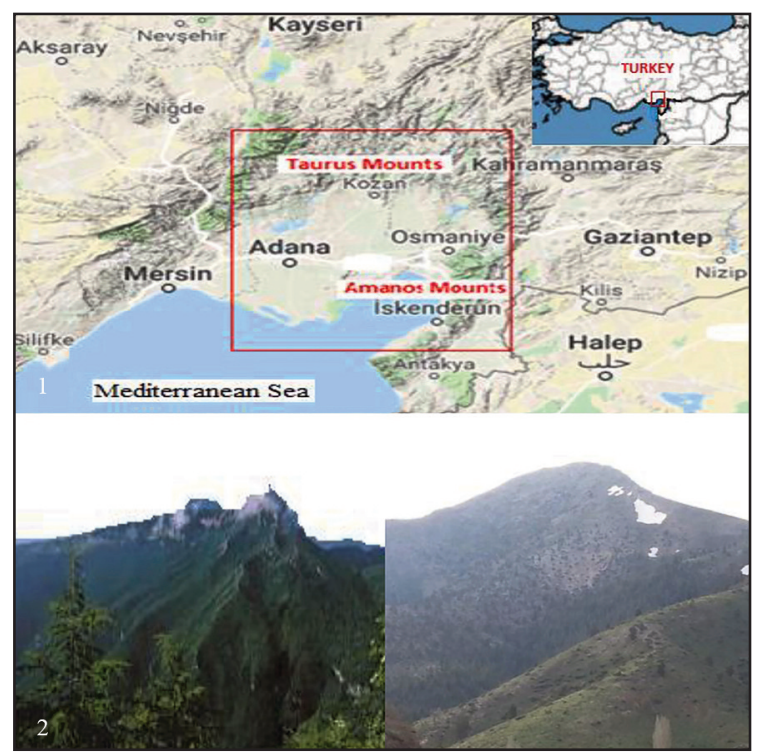

Figure 1, 2. Study area in Turkey's eastern Mediterranean Region. Fig. 1: map. Fig. 2: general views of the study area. 
Low altitude parts of the research area (up to $1,200 \mathrm{~m}$ ) have a Mediterranean climate, with warm, dry summers and cool, wet winters. The high-altitude parts of the study area (between approximately $1,200 \mathrm{~m}$ and 2,495 $\mathrm{m}$ ) experience a continental climate, with cold, snowy, and rainy winters and cool, dry summers. Annual rainfall varies between 400 and 1,500 $\mathrm{mm}$, depending on the elevation and direction. Seasonal rainfall regime is as follows: winter, spring, autumn, and summer. From the beginning of December until the end of March, precipitation in the form of snow is seen at places above $1,000 \mathrm{~m}$. The average annual temperature of the area is $19.3{ }^{\circ} \mathrm{C}$. In August, average maximum temperature is $34.8^{\circ} \mathrm{C}$ and the minimum average temperature of $4.8^{\circ} \mathrm{C}$ is seen in January (Türkmen \& Düzenli, 1990; Öztürk et al., 2008; Özkan, 2008; Türkmen et al., 2015b).

\section{RESULTS AND DISCUSSION}

Our investigations on the specimens collected from the area and identified with the help of Flora of Turkey (Davis, 1965-1985; Davis et al., 1988; Güner et al., 2000) have revealed that 1,259 vascular plant taxa belonging to 469 genera from 106 families (Türkmen \& Düzenli, 1990; Türkmen \& Düzenli, 1998; Türkmen, 2014; Türkmen et al., 2015a, b). Author citiations of plant names followed Brummitt \& Powell (1992).

The phytogeographical distribution of these taxa is as follows (Table 1): Mediterranean: 328 (26.1\%), Irano-Turanian: 157 (12.5\%), Euro-Siberian: $87(6.8 \%)$, cosmopolitan and unknown: 687 $(54.6 \%)$. The total number of endemic species was 195 and the endemism rate was $15.1 \%$.

As seen in Table 1, plains (low altitudes terrains) usually have a uniform vegetation, so there are few plant species. On the other hand, mountains (steep terrains) are rich in vegetation because they create different environmental conditions.

The Mediterranean elements are dominating the list simply due to the prevalence of Mediterranean climate here. The Euro-Siberian elements, which were widely distributed in the Pleistocene ice age, have progressed southwards along the Anatolian Diagonal, and due to the humid climatic conditions, they are still continuing to grow in the research area. The Euro-Siberian floristic region in Turkey is a very widespread area with typical Fagus orientalis Lipsky forest formation. In the Amanos mountains, large, frequent, and pure community is distributed on the northern slopes between 1600 and 1900 m (Davis et al., 1971; Y1lmaz, 1993; Türkmen \& Düzenli, 1998).

The dominating families and the number of taxa these embody are: Asteraceae: 163 (12.95\%), Fabaceae: 132 (10.48\%), Lamiaceae: 87 (6.91\%), Brassicaceae: 85 (6.75\%), Poaceae: 81 (6.35\%), Liliaceae: 62 (4.92\%), Caryophyllaceae: 59 (4.68\%), Apiaceae: 50 (3.97\%), Rosaceae: 45 (3.57\%), Scrophulariaceae: 44 (3.49\%), Boraginaceae: 35 (2.78\%); Rubiaceae: 25 (1.99\%); Polygonaceae: 17 (1.35\%), Euphorbiaceae: 16 (1.27\%), Hypericaceae: 16 (1.27\%), Orchidaceae: 16 (1.27\%), Ranunculaceae: 15 (1.19\%), Crassulaceae: $12(0.95 \%)$, and Geraniceae: 12 (0.95\%).

The rare and endemic species and their International Union for Conservation of Nature (IUCN) categories are as follows: CR: 1, EN: 1, VU: 15, DD: 2, NT: 12, and LC: 22 (Table 2) (Ekim et al., 2000; IUCN, 2017).

The main types of vegetation observed in the research area are maquis, forest, steppe, and aquatic vegetations (Türkmen \& Düzenli, 1990; Türkmen et al. 2015a, b):

Shrub-like vegetation (macchia) is the dominant vegetation between $300 \mathrm{~m}$ and $600 \mathrm{~m}$, most of which are evergreen shrubs. Common species include Quercus coccifera L., Erica manipuliflora Salisb., Myrtus communis L. ssp. communis L., Cotinus coggygria Scop., Calicotome villosa (Poiret) Link., Phillyrea latifolia L., Olea europaea L. var. sylvestris (Mill.) Lehr, Pistacia terebinthus L. ssp. palaestina (Boiss.) Engl., Cistus creticus L., Rham-

\begin{tabular}{|l|c|c|c|c|c|c|}
\hline \multirow{2}{*}{$\begin{array}{l}\text { Floral } \\
\text { parameters }\end{array}$} & \multicolumn{2}{|c|}{ Number of species } & \multicolumn{3}{|c|}{ Percent of sample total } \\
\cline { 2 - 7 } & $\begin{array}{c}\text { Mount } \\
1\end{array}$ & $\begin{array}{c}\text { Plain } \\
1\end{array}$ & $\begin{array}{c}\text { Plain } \\
2\end{array}$ & $\begin{array}{c}\text { Mount } \\
1\end{array}$ & $\begin{array}{c}\text { Plain } \\
1\end{array}$ & $\begin{array}{c}\text { Plain } \\
2\end{array}$ \\
\hline $\begin{array}{l}\text { Mediterranean } \\
\text { elements }\end{array}$ & 328 & 151 & 73 & 26.1 & 36.4 & 17.5 \\
\hline $\begin{array}{l}\text { Euro-Siberian } \\
\text { elements }\end{array}$ & 157 & 11 & 7 & 12.5 & 2.7 & 3.9 \\
\hline $\begin{array}{l}\text { Irano-Turanian } \\
\text { elements }\end{array}$ & 87 & 13 & 6 & 6.8 & 3.1 & 3.4 \\
\hline $\begin{array}{l}\text { Multiregional } \\
\text { elements }\end{array}$ & 687 & 240 & & 54.6 & 57.8 & 51.7 \\
\hline Endemics & 195 & 20 & 8 & 15.1 & 4.8 & 4.5 \\
\hline Total flora & 1259 & 415 & 1178 & - & - & - \\
\hline
\end{tabular}

Table 1. A comparison of the floral characteristics in the research area and lowland areas (Mount 1 (present study); Plain 1 (Türkmen \& Düzenli, 1990); Plain 2 (Bulut, 2008). 


\begin{tabular}{|c|c|}
\hline Endangered plants & Conservation status \\
\hline $\begin{array}{l}\text { Acantholimon acerosum (Willd.) } \\
\text { boiss. var.. } \\
\text { brachystachyum Boiss. }\end{array}$ & $\mathrm{VU}(\mathrm{B} 1 \mathrm{a}, \mathrm{b}$ and $\mathrm{B} 2 \mathrm{a}, \mathrm{b})$ \\
\hline Acanthus dioscoridis L. var. laciniatus Freyn & NT \\
\hline $\begin{array}{l}\text { Acanthus dioscoridis L. var. perringii (Siehe) E.. } \\
\text { Hossain }\end{array}$ & $\mathrm{VU}(\mathrm{B} 1 \mathrm{a}, \mathrm{b}$ and B2 a,b) \\
\hline Achillea cappadocica Hauskn. et Bornm. & $\mathrm{LC}$ \\
\hline Achillea monocephala Boiss. et Balansa & $\mathrm{EN}(\mathrm{B} 1 \mathrm{a}, \mathrm{b}$ and $\mathrm{B} 2 \mathrm{a}, \mathrm{b})$ \\
\hline Aethionema capitatum Boiss. et Balansa & NT \\
\hline Aethionema schistosum Boiss. et Kotschy & NT \\
\hline Alcea apterocarpa (Fenzl) Boiss. & LC \\
\hline Alkanna amana Rech. fil. & $\mathrm{DD}$ \\
\hline Alkanna cappadocica Boiss. et Balansa & LC \\
\hline Alkanna kotschyana DC. & LC \\
\hline Allium brevicaule Boiss. et Balansa & NT \\
\hline Allium cappadocicum Boiss. & NT \\
\hline Allium glumaceum Boiss. et Hauskn. & NT \\
\hline Allium tauricola Boiss. & NT \\
\hline Allium tchihatschewii Boiss. & $\mathrm{LC}$ \\
\hline Alnus glutinosa (L.) Gaertn. ssp. antitaurica Yalt. & NT \\
\hline Alyssum argrophyllum Schott & NT \\
\hline Alyssum callichroum Boiss. et Balansa & $\mathrm{LC}$ \\
\hline Alyssum corningii Dudley & NT \\
\hline Alyssum huetii Boiss. & $\mathrm{LC}$ \\
\hline Alyssum masmenaeum Boiss. & $\mathrm{LC}$ \\
\hline $\begin{array}{l}\text { Alyssum murale Waldst. et Kit. var. haradjianii } \\
\text { (Rech.) Dudley }\end{array}$ & $\mathrm{VU}(\mathrm{B} 1 \mathrm{a}, \mathrm{b}$ and $\mathrm{B} 2 \mathrm{a}, \mathrm{b})$ \\
\hline Alyssum oxycarpum Boiss. et Balansa & NT \\
\hline $\begin{array}{l}\text { Alyssum peltarioides Boiss. ssp. virgatiforme } \\
\text { (Nyar.) Dudley }\end{array}$ & LC \\
\hline Alyssum praecox Boiss. et Balansa var. praecox & LC \\
\hline $\begin{array}{l}\text { Anchusa leptophylla Roemer et Schultes ssp. } \\
\text { incana }\end{array}$ & $\mathrm{LC}$ \\
\hline Anthemis aciphylla Boiss. var. aciphylla & $\mathrm{LC}$ \\
\hline $\begin{array}{l}\text { Arenaria ledebouriana Fenzl var. pauciflora } \\
\text { McNeill }\end{array}$ & LC \\
\hline Asperula cymulosa (Post) Post & $\mathrm{VU}(\mathrm{B} 1 \mathrm{a}, \mathrm{b}$ and $\mathrm{B} 2 \mathrm{a}, \mathrm{b})$ \\
\hline $\begin{array}{l}\text { Asperula stricta Boiss. ssp. grandiflora Schönb.- } \\
\text { Tem. }\end{array}$ & NT \\
\hline Asperula stricta Boiss. ssp. monticola Ehr & $\mathrm{LC}$ \\
\hline Asphodeline cilicica Tuzlacı & $\mathrm{VU}(\mathrm{B} 1 \mathrm{a}, \mathrm{b}$ and $\mathrm{B} 2 \mathrm{a}, \mathrm{b})$ \\
\hline Astragalus antiochimus Post & $\mathrm{DD}$ \\
\hline $\begin{array}{l}\text { Astragalus campylosema Boiss. ssp. atropurpureus } \\
\text { (Boiss.) Chamb. }\end{array}$ & LC \\
\hline Astragalus campylosema Boiss. ssp. campylosema & LC \\
\hline Astragalus condensatus Ledeb. & $\mathrm{LC}$ \\
\hline Astragalus globosus Vahl & $\mathrm{LC}$ \\
\hline Aubrieta canescens (Boiss.) Bornm. ssp. canescens & $\mathrm{LC}$ \\
\hline $\begin{array}{llll}\text { Aubretia canescens (Boiss.) } & \text { Bornm. } & \text { ssp. } \\
\text { macrostyla } \text { Cullen et Huber-Morat } & & \end{array}$ & $\mathrm{LC}$ \\
\hline Ballota macrodonta Boiss. et Balansa & $\mathrm{VU}(\mathrm{B} 1 \mathrm{a}, \mathrm{b}$ and $\mathrm{B} 2$ \\
\hline
\end{tabular}

\begin{tabular}{|c|c|}
\hline Endangered plants & Conservation status \\
\hline $\begin{array}{l}\text { Bolanthus minuartioides (Jaub. et Spach) Hub.- } \\
\text { Mor. }\end{array}$ & $\mathrm{LC}$ \\
\hline Bupleurum lophocarpum Boiss. et Balansa & NT \\
\hline \begin{tabular}{llll|} 
Carduus olympicus & Boiss. & ssp. & hypoleucus \\
(Bornm.) Davis & & & \\
\end{tabular} & $\mathrm{LC}$ \\
\hline $\begin{array}{l}\text { Carex divulsa Stokes ssp. coriogyne (Nelmes) } \\
\text { Ö.Nilsson }\end{array}$ & $\mathrm{LC}$ \\
\hline Centaurea aladaghensis Wagenitz & $\mathrm{EN}(\mathrm{B} 1 \mathrm{a}, \mathrm{b}$ and $\mathrm{B} 2 \mathrm{a}, \mathrm{b})$ \\
\hline Centaurea antitauri Hayek & $\mathrm{VU}(\mathrm{B} 1 \mathrm{a}, \mathrm{b}$ and $\mathrm{B} 2 \mathrm{a}, \mathrm{b})$ \\
\hline $\begin{array}{l}\text { Centaurea calcitrapa L. ssp. cilicica (Boiss. et } \\
\text { Balansa) Wagenitz }\end{array}$ & NT \\
\hline Centaurea cataonica Boiss. et Hausskn. & NT \\
\hline Centaurea haussknechtii Boiss. & $\mathrm{CR}(\mathrm{B} 1 \mathrm{a}, \mathrm{b}$ and $\mathrm{B} 2 \mathrm{a}, \mathrm{b})$ \\
\hline Centaurea lycopifolia Boiss. et Kotschy & NT \\
\hline Centaurea mucronifera DC. & $\mathrm{LC}$ \\
\hline Centaurea ptosimopappoides Wagenitz & $\mathrm{VU}(\mathrm{B} 1 \mathrm{a}, \mathrm{b}$ and $\mathrm{B} 2 \mathrm{a}, \mathrm{b})$ \\
\hline Cephalaria taurica Szabo & $\mathrm{VU}(\mathrm{B} 1 \mathrm{a}, \mathrm{b}$ and $\mathrm{B} 2 \mathrm{a}, \mathrm{b})$ \\
\hline $\begin{array}{l}\text { Chaenorhinum litorale (Bernh.) Fritsch } \text { ssp. } \\
\text { pterosporum (Fisch. et C.A.Mey.) Davis }\end{array}$ & $\mathrm{LC}$ \\
\hline Chamaecytisus drepanolobus (Boiss.) Rothman & NT \\
\hline Cicer echinospermum P.H.Davis & $\mathrm{LC}$ \\
\hline Cirsium ellenbergii Bornm. & $\mathrm{VU}(\mathrm{B} 1 \mathrm{a}, \mathrm{b}$ and $\mathrm{B} 2 \mathrm{a}, \mathrm{b})$ \\
\hline Cochleria sempervivum Boiss. et Balansa & NT \\
\hline Crepis armena DC. & $\mathrm{LC}$ \\
\hline Crepis macropus Boiss. et Heldr. & $\mathrm{LC}$ \\
\hline Crocus cancellatus Herb. ssp. cancellatus & $\mathrm{LC}$ \\
\hline $\begin{array}{l}\text { Crocus kotschyanus C. Koch ssp. cappadocicus } \\
\text { Mathew }\end{array}$ & NT \\
\hline Cyclamen pseud-ibericum Hildebr. & $\mathrm{EN}(\mathrm{B} 1 \mathrm{a}, \mathrm{b}$ and $\mathrm{B} 2 \mathrm{a}, \mathrm{b})$ \\
\hline Dactylorhiza osmanica (L.) Soo' var. osmanica & $\mathrm{LC}$ \\
\hline Dianthus balansae Boiss. & $\mathrm{LC}$ \\
\hline Dianthus brevicaulis Fenzl ssp. brevicaulis & $\mathrm{LC}$ \\
\hline Dianthus lactiflorus Fenzl & NT \\
\hline Ebenus laguroides Boiss. var. laguroides & $\mathrm{LC}$ \\
\hline Eryngium kotschyi Boiss. & $\mathrm{LC}$ \\
\hline Erysimum alpestre Kotschy ex Boiss. & $\mathrm{LC}$ \\
\hline Erysimum leptocarpum Gay & EN (B1 a,b and B2 a,b) \\
\hline Erysimum sintenisianum Bornm. & $\mathrm{LC}$ \\
\hline Euphorbia cardiophylla Boiss. et Heldr. & $\mathrm{LC}$ \\
\hline Euphorbia rhytidosperma Boiss. et Balansa & $\mathrm{VU}(\mathrm{B} 1 \mathrm{a}, \mathrm{b}$ and $\mathrm{B} 2 \mathrm{a}, \mathrm{b})$ \\
\hline Ferula drudeana Korovin & $\mathrm{VU}(\mathrm{B} 1 \mathrm{a}, \mathrm{b}$ and $\mathrm{B} 2 \mathrm{a}, \mathrm{b})$ \\
\hline Ferulago pachyloba Fenzl & $\mathrm{VU}(\mathrm{B} 1 \mathrm{a}, \mathrm{b}$ and $\mathrm{B} 2 \mathrm{a}, \mathrm{b})$ \\
\hline Festuca adanensis Markgr.-Dannenb. & NT \\
\hline Fraxinus ornus L. ssp. cilicica (Lingelsh.) Yalt. & $\mathrm{LC}$ \\
\hline Galatella amani (Post) Grierson & $\mathrm{VU}(\mathrm{B} 1 \mathrm{a}, \mathrm{b}$ and $\mathrm{B} 2 \mathrm{a}, \mathrm{b})$ \\
\hline Galium cilicicum Boiss. & $\mathrm{LC}$ \\
\hline Galium stepparum Ehrend. et Schönb.-Tem. & NT \\
\hline Gladiolus anatolicus (Boiss.) Stapf & $\mathrm{LC}$ \\
\hline Graellsia davisiana Poulter & EN (B1 a,b and B2 a,b) \\
\hline Haplophyllum myrtifolium Boiss. & $\mathrm{LC}$ \\
\hline
\end{tabular}




\begin{tabular}{|c|c|}
\hline Endangered plants & Conservation status \\
\hline $\begin{array}{l}\text { Helichrysum arenarium (L.) Moench ssp. aucheri } \\
\text { (Boiss.) Davis et Kupicha }\end{array}$ & LC \\
\hline Helleborus vesicarius Aucher & NT \\
\hline Herniaria argaea Boiss. & $\mathrm{VU}(\mathrm{B} 1 \mathrm{a}, \mathrm{b}$ and $\mathrm{B} 2 \mathrm{a}, \mathrm{b})$ \\
\hline Herniaria. saxatilis Brummitt & $\mathrm{VU}(\mathrm{B} 1 \mathrm{a}, \mathrm{b}$ and $\mathrm{B} 2 \mathrm{a}, \mathrm{b})$ \\
\hline $\begin{array}{l}\text { Hieracium lasiochaetum (Bornm. et Zahn) Sell et } \\
\text { West }\end{array}$ & LC \\
\hline Hyacinthella micrantha (Boiss.) Chouard & NT \\
\hline $\begin{array}{|lllll|}\text { Hyacinthus } & \text { orientalis } & \text { L. } & \text { ssp. } & \text { chionophilus } \\
\text { Wendelbo } & & & & \\
\end{array}$ & NT \\
\hline $\begin{array}{l}\text { Hypericum aviculariifolium Jaub. et Spach ssp. } \\
\text { aviculariifolium var. aviculariifolium }\end{array}$ & NT \\
\hline $\begin{array}{l}\text { Hypericum avicularifolium Jaub. et Spach ssp. } \\
\text { depilatum (Freyn et Bornm.) Robson var. depilatum }\end{array}$ & LC \\
\hline Hypericum kotschyanum Boiss. & NT \\
\hline $\begin{array}{l}\text { Hypericum. lanuginosum Lam. var. scabrellum } \\
\text { (Boiss.) Robson }\end{array}$ & $\mathrm{LC}$ \\
\hline Inula anatolica Boiss. & LC \\
\hline Isatis candolleana Boiss. & $\mathrm{LC}$ \\
\hline Isatis constricta P.H.Davis & EN (B1 a,b and B2 a,b) \\
\hline Isatis floribunda Boiss. ex Bornm. & LC \\
\hline \begin{tabular}{|l|} 
Johrenia silenoides Boiss. et Balansa \\
\end{tabular} & NT \\
\hline $\begin{array}{l}\text { Juncus sparganiifolius Boiss. et Kotschy ex } \\
\text { Buchenau }\end{array}$ & $\mathrm{LC}$ \\
\hline Kundmannia syriaca Boiss & LC \\
\hline Lamium microphyllum Boiss. & $\mathrm{VU}(\mathrm{B} 1 \mathrm{a}, \mathrm{b}$ and $\mathrm{B} 2 \mathrm{a}, \mathrm{b})$ \\
\hline $\begin{array}{l}\text { Lamium garganicum L. ssp. nepetifolium (Boiss.) } \\
\text { R.R.Mill }\end{array}$ & LC \\
\hline Lathyrus elongatus (Bornm.) Sirj. & NT \\
\hline $\begin{array}{|llll|}\text { Lathyrus. laxiflorus } \quad \text { (Desf.) } & \text { Kuntze } & \text { ssp. } \\
\text { angustifolius } & \text { (Post ex Dinsm.) Davis }\end{array}$ & $\mathrm{VU}(\mathrm{B} 1 \mathrm{a}, \mathrm{b}$ and $\mathrm{B} 2 \mathrm{a}, \mathrm{b})$ \\
\hline $\begin{array}{l}\text { Leucocyclus formosus Boiss. ssp. amanicus (Rech. } \\
\text { fil.) Hub.-Mor. et Grierson }\end{array}$ & NT \\
\hline $\begin{array}{l}\text { Linaria genistifolia (L.) Mill. ssp. confertiflora } \\
\text { (Boiss.) Davis }\end{array}$ & LC \\
\hline $\begin{array}{l}\text { Marrubium globosum Montbret et Aucher ex } \\
\text { Benth, ssp. globosum }\end{array}$ & LC \\
\hline Michauxia tchihatchewii Fisch. et C.A.Mey. & NT \\
\hline Micromeria cilicica Hauskn. ex P.H.Davis & EN (B1 a,b and B2 a,b) \\
\hline $\begin{array}{l}\text { Micromeria cremnophila Boiss. et Heldr. ssp. } \\
\text { amana (Rech..f.) P.H.Davis }\end{array}$ & LC \\
\hline $\begin{array}{l}\text { Micromeria cremnophila Boiss. et Heldr. ssp. } \\
\text { anatolica } \text { P.H.Davis }\end{array}$ & $\mathrm{LC}$ \\
\hline Minuartia tchihatchewii (Boiss.) Hand.-Mazz. & LC \\
\hline $\begin{array}{l}\text { Minuartia umbellulifera (Boiss.) McNeill ssp. } \\
\text { umbellulifera var. umbellulifera }\end{array}$ & $\mathrm{LC}$ \\
\hline Muscari aucheri (Boiss.) Baker & LC \\
\hline Muscari azureum Fenzl & LC \\
\hline Muscari bourgaei Baker & $\mathrm{LC}$ \\
\hline Muscari microstomum Davis et Stuart & $\mathrm{VU}(\mathrm{B} 1 \mathrm{a}, \mathrm{b}$ and $\mathrm{B} 2 \mathrm{a}, \mathrm{b})$ \\
\hline
\end{tabular}

\begin{tabular}{|c|c|}
\hline Endangered plants & Conservation status \\
\hline Nepeta isaurica Boiss. et Heldr. apud Benth. & LC \\
\hline Onobrychis armena Boiss. et A.Huet & LC \\
\hline Onosma armenum DC. & LC \\
\hline Onosma bracteosum Hauskn. et Bornm. & LC \\
\hline Onosma inexspectatum Teppner & LC \\
\hline Ornithogalum alpigenum Stapf & NT \\
\hline Papaver triniifolium Boiss. & LC \\
\hline Paronychia argyroloba Stapf & NT \\
\hline Paronychia chionaea Boiss. & EN (B1 a,b and B2 a,b) \\
\hline Paronychia mughlaei Chaudhuri & $\mathrm{VU}(\mathrm{B} 1 \mathrm{a}, \mathrm{b}$ and $\mathrm{B} 2 \mathrm{a}, \mathrm{b})$ \\
\hline Phlomis armeniaca Willd. & LC \\
\hline $\begin{array}{l}\text { Phlomis longifolia Boiss. et Bal. var. bailanica } \\
\text { (Vierh.) Hub.-Mor. }\end{array}$ & NT \\
\hline $\begin{array}{l}\text { Phryna ortegioides (Fisch. et C.A.Mey.) Pax. et } \\
\text { Hoffm. }\end{array}$ & NT \\
\hline Potentilla calycina Boiss. et Balansa & LC \\
\hline Potentilla pulvinaris Fenzl & EN (B1 a,b and B2 a,b) \\
\hline Pterocephalus pinardii Boiss. & LC \\
\hline $\begin{array}{l}\text { Ptilostemon afer (Jacq) Greuter ssp. eburneus } \\
\text { Greuter }\end{array}$ & LC \\
\hline $\begin{array}{l}\text { Quercus petraea (Mat.) Liebl. ssp. pinnatiloba (C. } \\
\text { Koch) Menitsby }\end{array}$ & LC \\
\hline Rhamnus hirtellus Boiss. & LC \\
\hline Rhammus pichleri Schneider et Bornm. ex Bornm & NT \\
\hline Ricotia sinuata Boiss. et Heldr. & $\mathrm{LC}$ \\
\hline Rindera caespitosa (A. DC.) Bunge & LC \\
\hline Rosularia chrysantha (Boiss.) Tahkt. & LC \\
\hline Rosularia globulariifolia (Fenzl) Berge & LC \\
\hline Rumex gracilescens Rech. & NT \\
\hline Salvia cryptantha Montbret et Aucher ex Benth. & LC \\
\hline Salvia recognita Fisch. et C.A.Mey. & LC \\
\hline Saponaria prostrata Willd. ssp. prostrata & LC \\
\hline Satureja amani P.H. Davis & $\mathrm{CR}(\mathrm{B} 1 \mathrm{a}, \mathrm{b}$ and $\mathrm{B} 2 \mathrm{a}, \mathrm{b})$ \\
\hline Satureja cilicica P.H.Davis & NT \\
\hline Scabiosa kurdica Post & $\mathrm{VU}(\mathrm{B} 1 \mathrm{a}, \mathrm{b}$ and $\mathrm{B} 2 \mathrm{a}, \mathrm{b})$ \\
\hline Scorzonera semicana DC. & $\mathrm{LC}$ \\
\hline Scorzonera tomentosa $\mathrm{L}$. & LC \\
\hline Scutulleria glaphyrostachys Rech. fil. & $\mathrm{VU}(\mathrm{B} 1 \mathrm{a}, \mathrm{b}$ and $\mathrm{B} 2 \mathrm{a}, \mathrm{b})$ \\
\hline $\begin{array}{l}\text { Scutulleria rubicunda Hormem ssp. brevibracteata } \\
\text { (Stapf.) Edmond }\end{array}$ & LC \\
\hline Scutellaria salviifolia Benth & LC \\
\hline Senecio farfarifolius Boiss. et Kotschy & $\mathrm{LC}$ \\
\hline Senecio jurineifolius Boiss. et Balansa & $\mathrm{LC}$ \\
\hline Sideritis cilicica Boiss. et Bal. & $\mathrm{EN}(\mathrm{B} 1 \mathrm{a}, \mathrm{b}$ and $\mathrm{B} 2 \mathrm{a}, \mathrm{b})$ \\
\hline $\begin{array}{l}\text { Sideritis libanotica Labill. ssp. linearis (Benth.) } \\
\text { Bornm. }\end{array}$ & LC \\
\hline Sideritis phlomoides Boiss.et Balansa & NT \\
\hline Silene delicatula Boiss. ssp. delicatula & $\mathrm{LC}$ \\
\hline Stachys longiflora Boiss. et Balansa & DD \\
\hline Stachys rupestris Montbret et Aucher ex Benth. & $\mathrm{LC}$ \\
\hline
\end{tabular}




\begin{tabular}{|c|c|}
\hline Endangered plants & Conservation status \\
\hline Stachys sparsipilosa Bhattacharjee et Hub.-Mor. & $\mathrm{LC}$ \\
\hline $\begin{array}{lllll}\text { Tanacetum argenteum } & \text { (Lam.) } & \text { Willd. } & \text { ssp. } \\
\text { argenteum } & & & & \\
\end{array}$ & LC \\
\hline $\begin{array}{l}\text { Tanacetum densum (Labill.) Schultz Bip. ssp. } \\
\text { amani Heywood }\end{array}$ & LC \\
\hline $\begin{array}{l}\text { Tanacetum densum (Labill.) Schultz Bip. ssp. } \\
\text { sivasicum Hub.-Mor. et Grierson }\end{array}$ & $\mathrm{VU}(\mathrm{B} 1 \mathrm{a}, \mathrm{b}$ and $\mathrm{B} 2 \mathrm{a}, \mathrm{b})$ \\
\hline Tanacetum haradjanii (Rech.f.) Grierson & NT \\
\hline Teucrium chamaedrys L. ssp. tauricolum Rech.f. & $\mathrm{LC}$ \\
\hline Thesium cilicicum Bornm. & NT \\
\hline Thlaspi densiflorum Boiss. et Kotschy & NT \\
\hline Thlaspi rosulare Boiss. et Balansa & $\mathrm{CR}(\mathrm{B} 1 \mathrm{a}, \mathrm{b}$ and $\mathrm{B} 2 \mathrm{a}, \mathrm{b})$ \\
\hline Thlaspi violascens Boiss. & $\mathrm{LC}$ \\
\hline Thymus sipyleus Boiss. ssp. sipyleus var. sipyleus & NT \\
\hline $\begin{array}{l}\text { Tordylium elegans (Boiss. et Balansa) Alava et } \\
\text { Hub.-Mor. }\end{array}$ & NT \\
\hline Trifolium davisii E.Hossain & $\mathrm{VU}(\mathrm{B} 1 \mathrm{a}, \mathrm{b}$ and $\mathrm{B} 2 \mathrm{a}, \mathrm{b})$ \\
\hline Tripleurospermum fissurale (Sosn.) E.Hossain & NT \\
\hline Tulipa armena Boiss. var. lycica (Baker) Marais & $\mathrm{LC}$ \\
\hline Verbascum antitauricum Hub.-Mor. & $\mathrm{VU}(\mathrm{B} 1 \mathrm{a}, \mathrm{b}$ and $\mathrm{B} 2 \mathrm{a}, \mathrm{b})$ \\
\hline Verbascum caesareum Boiss. & $\mathrm{VU}(\mathrm{B} 1 \mathrm{a}, \mathrm{b}$ and $\mathrm{B} 2 \mathrm{a}, \mathrm{b})$ \\
\hline $\begin{array}{l}\text { Verbascum cheiranthifolium Boiss. var. asperilum } \\
\text { (Boiss.) Murb. }\end{array}$ & LC \\
\hline Verbascum cilicicum Boiss. & $\mathrm{VU}(\mathrm{B} 1 \mathrm{a}, \mathrm{b}$ and B2 a,b) \\
\hline Verbascum leianthoides Murb. & $\mathrm{VU}(\mathrm{B} 1 \mathrm{a}, \mathrm{b}$ and $\mathrm{B} 2 \mathrm{a}, \mathrm{b})$ \\
\hline Verbascum leianthum Benth. & NT \\
\hline Verbascum linearilobium (Boiss.) Hub.-Mor. & EN (B1 a,b and B2 a,b) \\
\hline Verbascum lyratifolium Koch ex Benth. & NT \\
\hline Verbascum protractum Fenzl ex Tchih. & NT \\
\hline Veronica balansae Stroh & $\mathrm{LC}$ \\
\hline Veronica cinerea Boiss. et Balansa & $\mathrm{LC}$ \\
\hline Veronica cuneifolia Don ssp. Cuneifolia & LC \\
\hline Veronica dichrus Schott et Kotschy & NT \\
\hline $\begin{array}{l}\text { Veronica hispidula Boiss. et A.Huet ssp. ixodes } \\
\text { (Boiss.et Balansa) M.A.Fisch. }\end{array}$ & NT \\
\hline $\begin{array}{l}\text { Veronica orientalis Boiss. Miller ssp. nimordi } \\
\text { (Reichter ex Stapf.) M.A. Fischer }\end{array}$ & $\mathrm{LC}$ \\
\hline Vicia canescens Lab. ssp. canescens & NT \\
\hline
\end{tabular}

Table 3. The endangered species of the study area and their IUCN red data list categories. *CR (B1 a,b and B2 a,b) Critically endangered: Extent of occurrence less than $5000 \mathrm{~km}^{2}$; area of occupancy less than $500 \mathrm{~km}^{2}$; known no more than five locations; inferred decline in the area, extent and/or quality of habitat. EN (B1 a, b and B2 a, b) Endangered: Extent of occurrence less than $100 \mathrm{~km}^{2}$; area of occupancy less than $10 \mathrm{~km}^{2}$; known to exist at only a single location; inferred decline in the area, extent and/or quality of habitat. VU (B1 a, b and B2 a, b) Vulnerable: Extent of occurrence less than $20,000 \mathrm{~km}^{2}$; area of occupancy less than $2000 \mathrm{~km}^{2}$; known no more than ten locations; inferred decline in the area, extent, and/or quality of habitat. NT Near threatened. LC Least concern. DD Data deficient. nus oleoides L. ssp. graecus (Boiss et Reut.) Holmboe, R. punctatus Boiss. var. angustifolius Post, Arbutus andrachne L., Daphne sericea Vahl, Fontanesia philliraeoides Labill. ssp. philliraeoides Labill., and Styrax officinalis L.

Forest vegetation, from $300 \mathrm{~m}$ to $1900 \mathrm{~m}$, occupies different zones depending on local climate conditions, altitude, and topographic position. The common tree species in this belt are: Pinus brutia Ten., P. nigra Arn. ssp. pallasiana (Lamb.) Holmboe, Cedrus libani A. Rich., Abies cilicica (Ant. et Kotschy) Carr. ssp. cilicica (Ant. et Kotschy) Carr., Fagus orientalis Lipsky, Quercus cerris L. var. cerris L., and Carpinus orientalis Mill.. These tree species occur in mono and mixed stands, as herbaceous and shrub layer cover is weak. Native forests of pine, cedar, and cilician fir have been overlogged and replaced with Cistus laurifolius L., Juniperus excelsa M. Bieb., J. foetidissima Willd., J. drupaceae Labill., and J. oxycedrus L. ssp. oxycedrus L.

Steppe vegetation consisting of perennial herbaceous and semi-woody dwarf plants dominate the elevation zone above $1900 \mathrm{~m}$. These sparsely vegetated areas are covered by snow in winter until the middle of spring when herbaceous plants start emerging. The risk of landslides, floods, and avalanches increases due to overgrazing. The dominant species of this plant belt are: Acantholimon libanoticum Boiss., Ac. armenum Boiss. et A. Huet. var. armenum, Ac. venustum Boiss. et A. Huet var. armenum, Allium tauricola Boiss., Asphodelina damascene Boiss. et Baker ssp. damascene Boiss. et Baker, Asperula stricta Boiss. ssp. monticola Boiss., Astragalus angustifolius Lam. ssp. angustifolius Lam. var. angustifolius Lam., As. globosus Vahl, As. gummifer Labill., As. macrourus Fisch. et C.A.Mey., Berberis crataegyna DC., Cerasus prostrate (Labill.) Ser. var. prostrata (Labill.) Ser., Convolvulus compactus Boiss., Cotoneaster nummularia Fisch. et C.A.Mey., Echinops ritro L., Daphne oleoides Schreb. ssp. kurdica (Bornm.) Bornm., Echinops ritro L., Eremurus spectabilis Bieb., Euphorbia kotschyana Fenzl., Ferula elaeochytris Korovin, Haplophyllum myrtifolium Boiss., Helichrysum arenarium (L.) Moench ssp. aucheri (Boiss.) Davis et Kupicha, Marrubium globosum Montbret et Aucher ex Benth. ssp. globosum, M. parviflorum Fisch. et C.A. Mey. ssp. parviflorum, Minuartia juniperina (L.) Maire et Petitm., Onobrychis cornuta (L.) Desv, Phlomis cap- 
itata Boiss., Potentilla pulvinaris Fenzl, Pterocephalus pinardii Boiss., Rosa foetida J. Herm., R. pulverulenta M. Bieb., Thymus kotschyanus Boiss et Hohen var. glabrescens Boiss. et Hohen., Verbascum amanum Boiss., and Vincetoxicum tmoleum Boiss.

Wetland vegetation is encountered only at creek banks and spring mouths and includes the herbaceous or woody aquatic plants such as Alchemilla pseudocartilinica Juz., Alopecurus arundinaceus Poir., Alnus glutinosa (L.) Gaertn. ssp. antitaurica Yalt., Barbarea minor C. Koch var. eriopoda Busch, Carex divulsa Stokes ssp. divulsa (Nelmes) O. Nilsson, Cirsium arvense (L.) Scop. ssp. vestitum (Wimmer et Grab.) Petrak, Cyperus longus L., Datisca cannabina L., Equisetum arvense L., Hypericum venustum Fenzl, Juncus inflexus L., Lysimachia vulgaris L., Lythrum salicaria L., Nasturtium officinale R.Br., Orchis laxiflora Lam., Pedicularis comosa L. var. acmodonta (Boiss.) Boiss., Phragmites australis (Cav.) Trin. ex Steudel, Platanus orientalis L., Prunella vulgaris L., Ranunculus kotschyi Boiss., Rubus canescens DC. var. glabratus (Godr.) Davis et Meikle, Salix excelsa J.F. Gmelin, Scirpoides holoschoenus (L.) Sojak, Setaria viridis (L.) P. Beauv., Sium sisarum L. var. lancifolium (M. Bieb) Thell., and Tamarix smyrnensis Bunge.

Human activities such as dams, new road constructions, settlements, excessive grazing, illegal cutting, forest fires, mining, and summer camps are threats to the vegetation in the study area.

\section{CONCLUSIONS}

Geomorphological units are one of the most important elements limiting living habitats in nature. Areas of high biodiversity are concentrated on specific geomorphological units.

Rugged topography causes a wide variety of ecological conditions. The plant varieties that adapt themselves to these environmental conditions are also diversified. The research area is very important because it has biological diversity, pharmacologi$\mathrm{cal} /$ industrial plant species, and recreation functions.

The high and rugged topography of the study area has contributed significantly to plant diversity and distribution. Large mountainous terrain leads to variability in climatic and lithological conditions. This is reflected in the plant cover. If the field in- vestigated from the other side had formed a smoother physical surface, a plant richness could not be mentioned on this scale.

The Anatolian Diagonal, which forms the connection between the northern and southern Anatolian mountains in the formation of endemism and species richness, played an important role in the plant movement in the glacial periods and glacial warming periods by acting as a bridge for some species while creating physical obstacles for some other species (Türkmen \& Düzenli, 1998; Atalay, 2006; Türkmen et al., 2015b)

In the near future, the climate change that will emerge in the region will undermine sustainable development efforts as well as affecting the phenological behaviors and living conditions of existing plant species, causing deterioration problems, especially in altitude along the Taurus and Amanos Mountains. These include the addition of anthropogenic activities such as illegal cutting, harvesting, settlement, land-clearing, overgrazing, and fire, which will inevitably change the largely preserved natural vegetation cover

Estimated climate scenarios for temperature increases will seriously threaten the life of the cryohygrophilous high-altitude plant taxon in the research area, interfering with hydrological conditions. Endemics will take full share of all these effects. Upward migrations due to warming temperatures will result in displacements in the vegetation belt here and drought-tolerant invaders from low altitudes will eventually lead to biodiversity losses (Öztürk et al., 2008; Güleryüz et al., 2010).

Mountain biodiversity faces serious and growing challenges.

The degradation of the habitat caused by nonsustainable land cleansment leads to the erosion of fertile soils and increases the avalanche, landslide, and flood threats. With this change in the habitat, rare plant and animal species can be depleted.

There are many challenges to overcome in order to use mountain biodiversity successfully and sustainably. The growing demand for water, the consequences of global climate change, tourism growth, and globalization are just a few of these challenges in the world, where the pressures of the industry and agriculture are on the rise.

Important threats of mountain ecosystems are: fire; climate change; land cover change, agricultural transformation, seismic hazards, infrastructure de- 
velopment, and armed conflict. These pressures disrupt mountain environments and affect the livelihoods of ecosystem services and people connected to them. In all mountain regions, natural risks are high and the effects of poor land-use practices are particularly intense. As ecosystems that represent the complex and integrated ecology of our planet, mountain environments are necessary for the survival of the global biosphere

Globalization is not only economic. It should also include the conservation and sustainable management of vegetation in all regions and other subregions around the world.

\section{REFERENCES}

Akman Y., 1973. Contribution a l'etude de la Flore les Montagnes de l'Amanus (I, II, III). Serie C, Communications de la Faculte des Sciences de l'Universite d'Ankara, 1-164.

Anonymous, 2010. Europe's Ecological Backbone: Recognising the True Value of Our Mountains. EEA (European Environment Agency) Report No 6, Copenhagen.

Anonymous, 2011. Tourism and Mountains. A Practical Guide to Good Practice, UNEP (United Nations Environment Programme).

Anonymous, 2018. Convention on biological diversity: What is mountain biodiversity? Available from: https://www.cbd.int/mountain/what.shtml

Atalay İ., 2002. Türkiye'nin Ekolojik Bölgeleri (Ecoregions of Turkey). T.C. Orman Bakanlığ AGM Yay. No:163 Meta Basımevi, İzmir.

Atalay İ., 2006. The Effects of Mountainous Areas on Biodiversity: A Case Study from the Northern Anatolian Mountains and the Taurus Mountains. Grazer Schriften der Geographie und Raumforschung, 41: 17-26.

Atay S., Güleryüz G., Orhun C., Seçmen Ö \& Vural C., 2009. Dağlarımızdaki Zenginlik Türkiye'nin 120 Alpin Bitkisi. Dönence Basım ve Yayın Hizmetleri, İstanbul.

Avcı M., 2005. Çeşitlilik ve Endemizm Açısından Türkiye'nin Bitki Örtüsü. İ.Ü. Ed. Fak. Coğrafya Bölümü, Coğrafya Dergisi, 13: 27-55.

Barrow M., 2018. The Mountain Environment. Available from:http://www.primaryhomeworkhelp.co.uk/ mountains.htm.

Baytop T., 2000. Anadolu Dağlarında 50 Yıl (Bir Bitki Avcısının Gözlemleri). Nobel Tip Kitapevleri, İstanbul, $480 \mathrm{pp}$.

Brummitt R.K. \& Powell C.E. (Eds.), 1992. Authors of plant names. Royal Botanic Gardens, Kew, London, $731 \mathrm{pp}$.
Bulut A., 2008. Hama Tepe, Deli Halil Tepe ve Tüysüz Tepe florasının belirlenmesi. Çıkurova Üniversitesi Fen Bilimleri Enstitüsü, Yüksek Lisans Tezi, Adana.

Davis P.H. (Ed.), 1965-1985. Flora of Turkey and the east Aegean Islands, vol. 1-9. Edinburgh University Press, Edinburgh.

Davis P.H., Harper P.C. \& Hedge I.C., 1971. Distribution patterns in Anatolia with particular reference to Endemizm. Plant Life SW Asia, Edinburgh; 15-27.

Davis P.H., Mill R.R. \& Tan K. (Eds.), 1988. Flora of Turkey and the east Aegean Islands. Vol 10 (Suppl. 1), Edinburgh University Press, Edinburgh.

Duran C., 2013. Türkiye'nin Bitki Çeşitliliğinde Dağlık Alanların Rolü. Biyoloji Bilimleri Araştırma Dergisi, 6: 72-77.

Eken G. \& Ataol M., 2011. Türkiye'nin Biyocoğrafyası. Available from: http://www.dogadernegi.org/turkiyenin-dogasi.aspx

Ekim T., Koyuncu M., Vural M., Duman H., Aytaç Z. \& Adigüzel N. (Eds.), 2000. Red data book of Turkish plants (Pteridophyta and Spermatophyta). Turkish Association for the Conservation of Nature Van Centennial University, Ankara, 246 pp.

Èrinç S., 1993. Türkiye Fiziki Coğrafyasının Ana Çizgileri. İ.Ü. Deniz Bilimleri ve Coğrafya Enstitüsü Bülteni, İstanbul, 10: 1-10.

Güleryuz G., Gücel S. \& Öztürk M., 2010. Nitrogen mineralization in a high altitude ecosystem in the Mediterranean phytogeographical region of Turkey. Journal of Environmental Biology, 31: 503-514.

Güner A., Özhatay N., Ekim T. \& Başer K.H.C. (Eds.), 2000. Flora of Turkey and the east Aegean Islands. Vol 11 (Suppl 2), Edinburgh University Press, Edinburgh, $656 \mathrm{pp}$.

IUCN., 2017. Guidelines for using the IUCN Red List Categories and Criteria: Version 13. Standards and Petitions Subcommittee of the IUCN Species Survival Commission. Available from: http://www.iucnredlist.org/documents/RedListGuidelines.pdf.

Kantarc1 M.D., 2005. Orman Ekosistemleri Bilgisi. İ.Ü. Orman Fakültesi Toprak İlmi ve Ekoloji Anabilim Dalı, İstanbul, 418 pp.

Karakuş H. \& Turkmen N., 2014. The floristic characteristics of plants in gardens and streets in urban areas of Adana, Turkey. Pakistan Journal of Botany, 46: 1935-1944

Kılınç M. \& Kutbay G., 2004. Bitki Ekolojisi. Plame Yayıncilık, Ankara.

Körner C., 1999. Alpine Plant Life: Functional Plant Ecology of High Mountain Ecosystems. Springer Verlag Berlin Heidelberg, Germany.

Özgül N., 1976. Toroslar'ın Bazı Temel Jeoloji Özellikleri, TJK Bülteni, Ankara, 19: 65-78.

Özkan K., 2008. Assessment to the relationships between vegetation and site properties accordance with simi- 
larity values between quadrate pairs. Biological Diversity and Conservation (BioDiCon), 1: 59-73.

Ozturk M., Celik A., Guvensen A. \& Hamzaoglu E., 2008. Ecology of tertiary relict endemic Liquidambar orientalis Mill forests. Forest Ecology Management, 256: $510-518$.

Pariona A., 2018. How Much Of The World's Land Mass Is Mountainous? Available from: https://www.worldatlas.com/articles/how-much-of-the-world-s-landmass-is-mountainous.html.

Sarı D., 2010. Biyoçeșitlilik ve floristik çeșitlilik açısından alpin alanların önemi. III. Ulusal Karadeniz Ormanc1lık Kongresi, 20-22 Mayıs, 4: 14471455.

Türkmen N., 2014. The Floristic Characteristics and Conservation Status of the Plant Species in the Tahtali Mountains, Turkey. Natural Areas Journal, 34: 111-119.

Türkmen N. \& Düzenli A., 1990. Doğu Akdeniz bölgesindeki korunmuș bir alanın doğal florası ve özel- likleri. Doga, Turkish Journal of Botany, 14: $97-$ 108.

Türkmen N. \& Düzenli A., 1998. The Flora of Dörtyol and Erzin Districts of Hatay Province of Turkey. Turkish Journal of Botany, 22: 121-141.

Türkmen N., Düzenli A., Karakuş H. \& Uma M.M., 2015a. Anthropogenic characteristics and conservation status of the vascular flora of Kozan Castle and its surrounding area (Turkey). Fresenius Environmental Bulletin, 24: 1189-1194.

Türkmen N., Düzenli A. \& Öztürk M., 2015b. Floral Diversity of the high altitudes of Amanos Mountains: A Case Study from Daz Mountain-Migır, Turkey. In: Ozturk M., Hakeem K.R., Faridah-Hanum I. \& Efe R. (Eds.), Climate Change Impacts on High-Altitude Ecosystems, Chapter, 17: 427-453.

Yılmaz K.T., 1993. Amanos Dağları Dörtyol Kesiminde Bazı Yayla Yerleşimlerinin Doğala Yakın Vejetasyonlar Üzerindeki Etkilerinin Araştırılması. Doktora Tezi, Ç.Ü. Fen Bilimleri Enstitüsü, Adana, 129 pp. 